\title{
Adrenocortical Adenoma and Central Serous Chorioretinopathy: A Rare Association?
}

\author{
S. Pastor-ldoate $\quad$ D. Peña ${ }^{a} \quad$ J.M. Herreras ${ }^{a, b}$

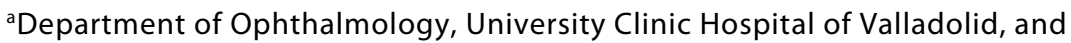 \\ bI.O.B.A, University of Valladolid, Valladolid, Spain
}

\section{Key Words}

Adrenocortical adenoma · Endogenous Cushing's syndrome $\cdot$ Central serous chorioretinopathy

\begin{abstract}
A 28-year-old man with bilateral central serous chorioretinopathy (CSCR) and body weight gain was diagnosed with Cushing's syndrome secondary to an adrenocortical adenoma. The patient had high levels of free cortisol and the tumor was confirmed by histopathology. After surgery, cortisol levels decreased and the CSCR spontaneously resolved. This case highlights the role of cortisol in the pathogenesis of CSCR. A diagnosis of endogenous Cushing's syndrome should be considered in patients with CSCR as an initial symptom.
\end{abstract}

\section{Introduction}

Central serous chorioretinopathy (CSCR) is a relatively frequent ocular disorder characterized by the accumulation of subretinal fluid at the posterior pole, causing a circumscribed area of retinal detachment [1]. The detachment frequently involves the macular area, such that patients usually complain of metamorphopsia, micropsia, macropsia, or central scotoma. Visual acuity is often moderately decreased and, in most cases, the CSCR spontaneously resolves within a few months. The pathophysiology remains unclear [1-6]. 


\section{Case Report}

A 28-year-old man presented at the Department of Ophthalmology, University Clinic Hospital of Valladolid, with a 3-month history of progressive bilateral vision loss, blurred vision, and metamorphopsia in both eyes. He denied any remarkable past medical history and concurrent systemic treatment; he also denied a history of abuse of tobacco, alcohol, or illegal drugs.

His best-corrected visual acuity was 20/25 in the right eye and 20/40 in the left eye. Anterior segment examination was normal in both eyes. Fundus oculi evaluation revealed retinal pigmentary abnormalities in the macula of both eyes ( $\underline{\mathrm{fig}}$. $1 \mathrm{a}, \mathrm{b}$ ). There was also a serous detachment of the neurosensory retina associated with a small retinal pigment epithelium (RPE) detachment in the left eye. The detachment was confirmed (fig. 1c, d) by optical coherence tomography (3D OCT-1000; Topcon, Tokyo, Japan). There was no evidence of intraocular inflammation, retinal holes, tears, or retinal choroidal neovascularization.

Autofluorescence images (TRC-50EX, Imaginet 2000; Topcon) showed diffuse areas of increased autofluorescence and reduced intensity in the macular and peripapillary areas. Upon fluorescein angiography, there were multiple pinpoint areas of hyperfluorescence in the macula and peripapillary areas. There were also areas of independent leakage with a mottled appearance and low activity from early stages without later changes (igg. 2). The patient was diagnosed with CSCR.

No specific treatment was prescribed for the CSCR, and 6 months later it remained unchanged. The patient's best-corrected visual acuity and fundus oculi evaluation revealed minimal changes. However, he suffered progressive systemic changes, including a 10-kg increase in body weight, supraclavicular fat accumulation around the neck and face (moon face), dorsocervical fat pad (buffalo hump), purple striae, and thin skin ( $\underline{\text { fig. }}$ 3a, b). A tentative diagnosis of hypercortisolism [7] was made, and the patient was sent to the Endocrinology Department of the University Clinic Hospital to confirm the diagnosis.

His urinary free cortisol was $264.7 \mu \mathrm{g} / 24 \mathrm{~h}$, confirming the tentative diagnosis of hypercortisolism. A low-dose (1 mg) dexamethasone suppression test was performed, revealing a mean cortisol level of $19.94 \mu \mathrm{g} / 100 \mathrm{ml}$. A follow-up high-dose $(8 \mathrm{mg})$ dexamethasone suppression test was performed, and a suppressed adrenocorticotropic hormone serum level assay value of $<5 \mathrm{pg} / \mathrm{ml}$ confirmed the previous dexamethasone suppression test. These results suggested the possibility of adrenocorticotropic hormone-independent Cushing's syndrome. Subsequently, an abdominal CT scan without contrast was performed and revealed the presence of a left adrenal oval-shaped mass with irregular enhancement (fig. $3 c, d$ ). Plasma aldosterone concentration, plasma renin activity, and 24-hour urinary catecholamines were negative.

Following these tests, the patient was treated with ketoconazole and steroid replacement therapy before consulting surgeons performed a laparoscopic left adrenalectomy. Histopathological examination revealed the presence of an adrenocortical adenoma. Hydrocortisone was administered after surgery to restore the physiologic hypothalamic-pituitary-adrenal axis.

One month after surgery, the patient's best-corrected visual acuity improved to 20/20 in both eyes. The fundus examination showed only RPE abnormalities, but there were no signs of CSCR. Optical coherence tomography images confirmed that the RPE detachment had resolved (fig. 1e, f).

\section{Discussion}

Psychological stress, hormonal changes in pregnancy, and glucocorticoid treatments [1-4] can all contribute to the development of CSCR, though the influence each of these circumstances has on CSCR is not well understood. Corticosteroids may inhibit collagen synthesis and increase choriocapillaris fragility and permeability by altering ion transport across the RPE [5]. Other proposed mechanisms of corticosteroid-dependent increases in capillary fragility and permeability include inhibition of fibroblast proliferation, alteration of leukocyte migration, and release of proteolytic enzymes $[4,5]$. Corticosteroids could 
also cause local ischemia by reducing choroidal fibrinolysis, leading to choroidal endothelial and RPE injury with consequent increased permeability [5].

There are few studies relating CSCR to endocrinological dysfunction characterized by hypercortisolism. CSCR has been reported in only $5 \%$ of patients with Cushing's syndrome, all of whom had a pituitary adenoma [6]. In each of these cases, the CSCR occurred during the period of active, untreated disease while plasma cortisol levels were high.

However, 2 new cases of CSCR have been reported as a result of hypercortisolism due to an adrenocortical adenoma [8]. Because both CSCR and adrenocortical adenomas are not very common diseases, a coincidental association is highly improbable. Furthermore, considering the resolution of CSCR in our patient without any systemic treatment following the adrenocortical adenoma resection, we suggest that hypercortisolism could be an important risk factor for CSCR. Our case underscores the importance of considering the possibility of a diagnosis of endogenous Cushing's syndrome in patients with CSCR as an initial symptom.

\section{Acknowledgement}

The authors thank M.I. López-Gálvez (Department of Ophthalmology, University Clinic Hospital of Valladolid, Spain) for providing the photography.

\section{Disclosure Statement}

The authors have no proprietary or financial interest concerning products or instruments described herein. 

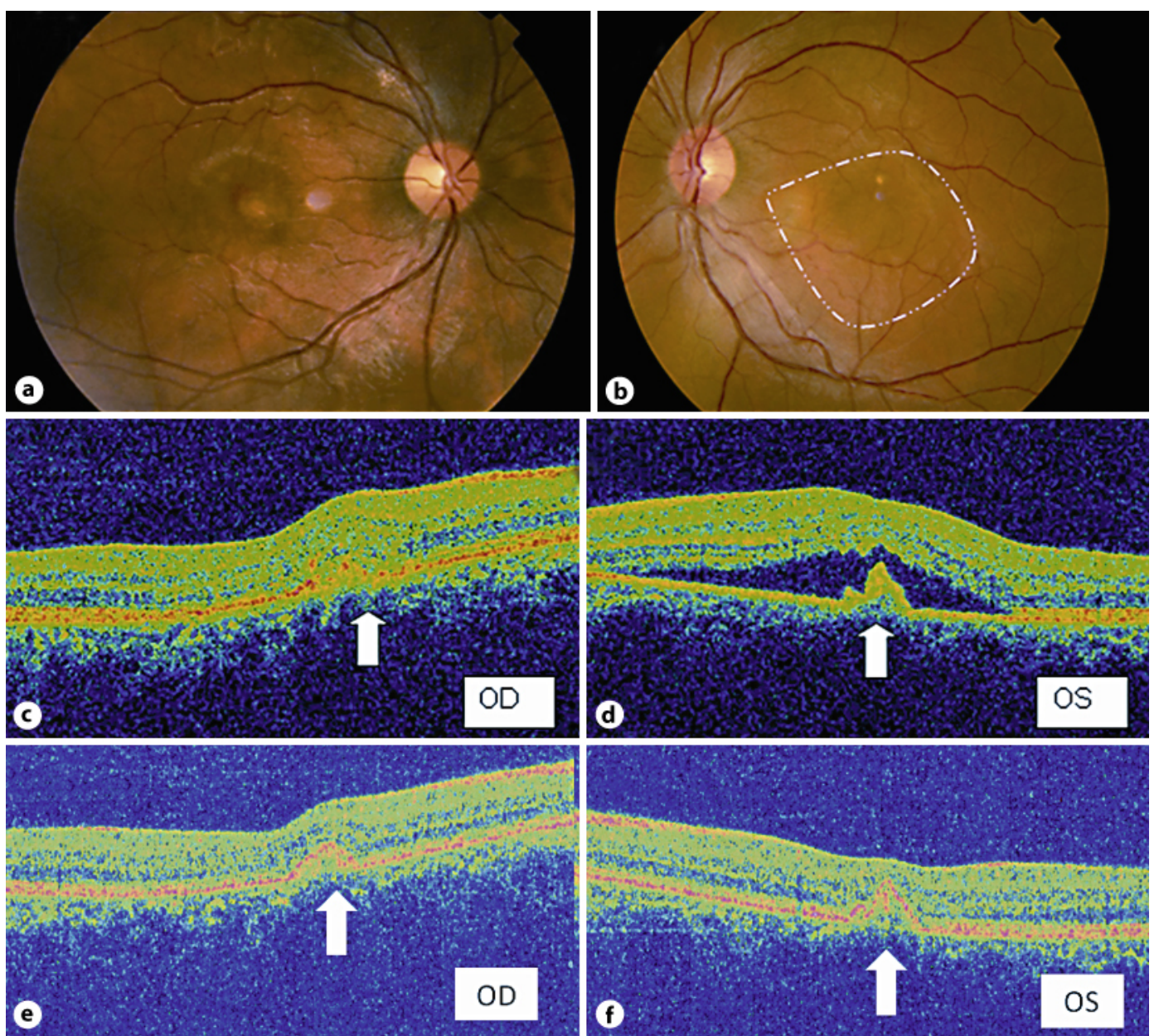

Fig. 1. Fundus color images of the right (a) and left (b) eye showing retinal pigmentary abnormalities in the macula and a serous retinal detachment in the left eye (white circumscribed area). Optical coherence tomography of the right (OD) (c) and left (OS) (d) eye showed chronic changes in the RPE layer (white arrows). The serous detachment of the neurosensory retina in the OS (d) was also evident. Optical coherence tomography images showed chronic changes in the RPE layer $(\mathbf{e}, \mathbf{f})$, and confirmed that the RPE detachment had resolved in the OS (f). 


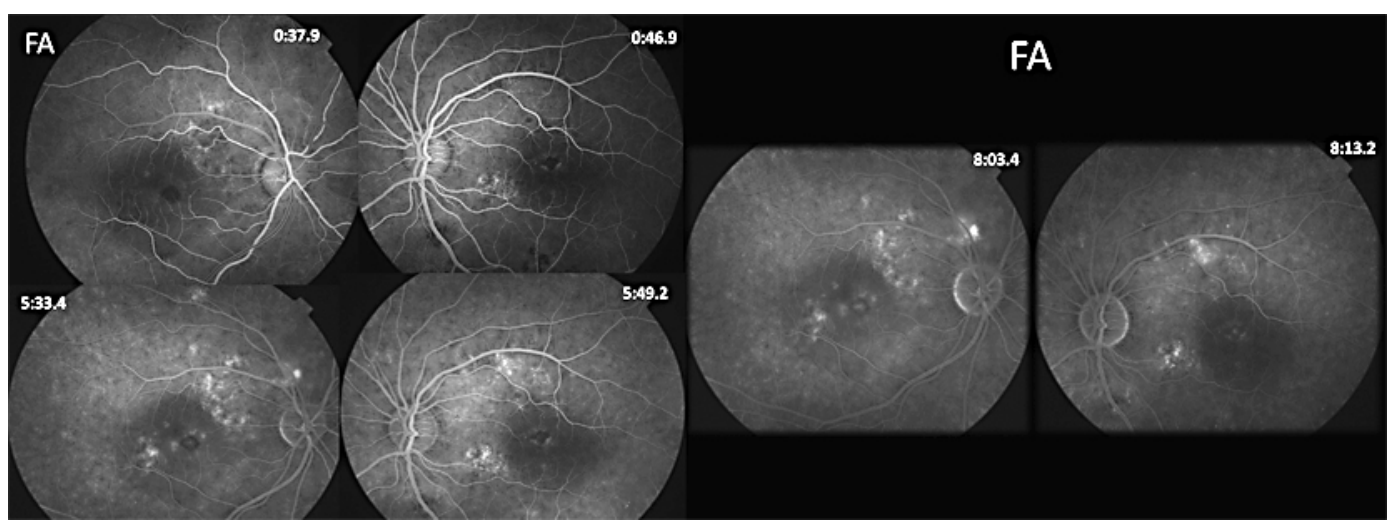

Fig. 2. Fluorescein angiography (FA) showed multiple pinpoint areas of hyperfluorescence in the macula and peripapillary areas. There was also independent leakage in areas with a mottled appearance and low activity from early stages.
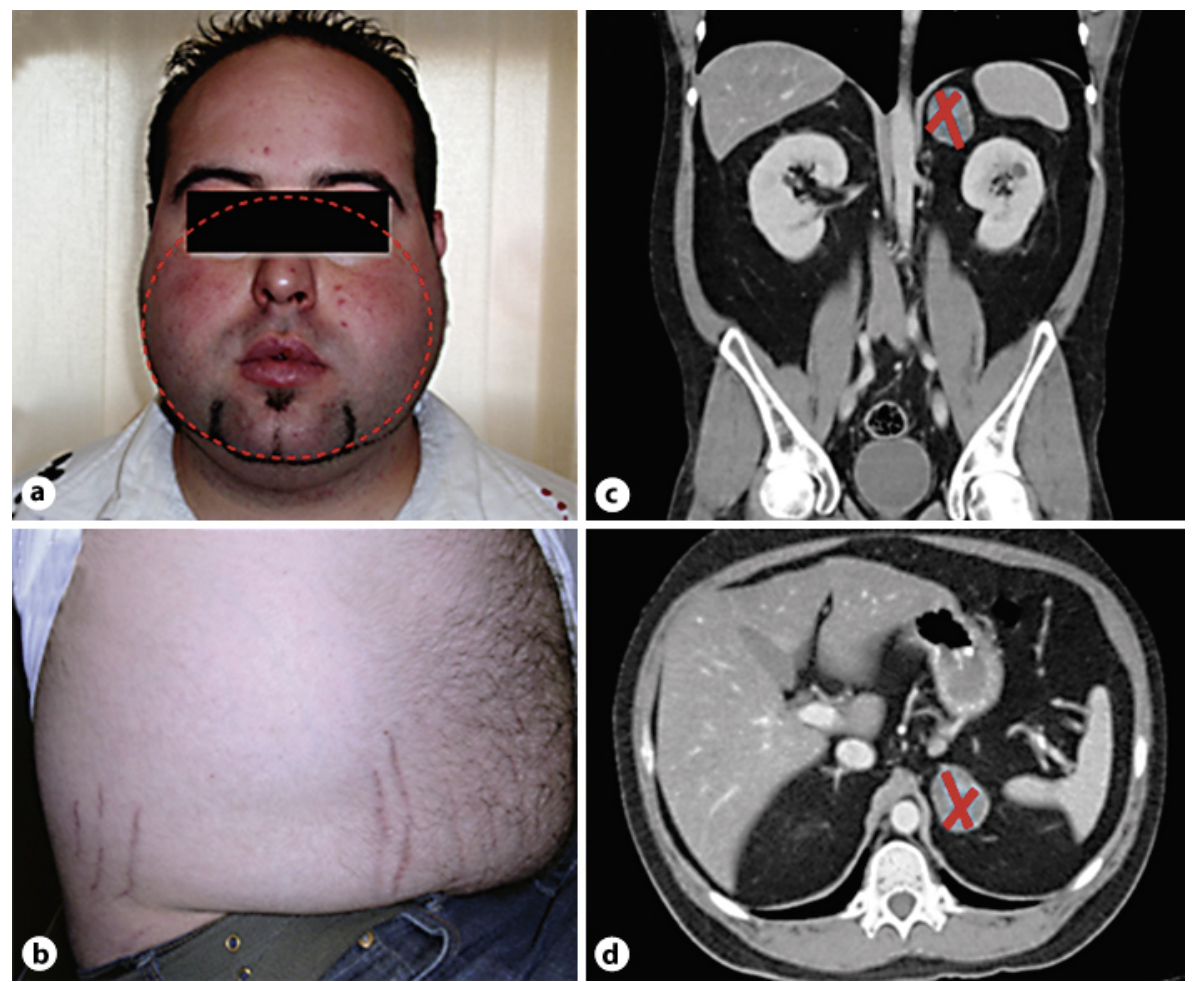

Fig. 3. The patient exhibited clinical features of endogenous Cushing's syndrome: moon face (a); overweight with purple striae on the skin (b). Abdominal CT scans revealed a left adrenal oval-shaped mass (red X) in coronal (c) and axial images $(\mathbf{d})$. 


\section{References}

1 Wang M, Munch IC, Hasler PW, Prunte C, Larsen M: Central serous chorioretinopathy. Acta Ophthalmol 2008;86:126-145.

2 Schultz KL, Birnbaum AD, Goldstein DA: Ocular disease in pregnancy. Curr Opin Ophthalmol 2005;16:308314.

3 Bouzas EA, Karadimas P, Pournaras CJ: Central serous chorioretinopathy and glucocorticoids. Surv Ophthalmol 2002;47:431-448.

-4 Carvalho-Recchia CA, Yannuzzi LA, Negrao S, Spaide RF, Freund KB, Rodriguez-Coleman H, Lenharo M, Iida T: Corticosteroids and central serous chorioretinopathy. Ophthalmology 2002;109:1834-1837.

-5 Levy J, Marcus M, Belfair N, Klemperer I, Lifshitz T: Central serous chorioretinopathy in patients receiving systemic corticosteroid therapy. Can J Ophthalmol 2005;40:217-221.

-6 Bouzas EA, Scott MH, Mastorakos G, Chrousos GP, Kaiser-Kupfer MI: Central serous chorioretinopathy in endogenous hypercortisolism. Arch Ophthalmol 1993;111:1229-1233.

-7 Nieman LK, Biller BM, Findling JW, Newell-Price J, Savage MO, Stewart PM, Montori VM: The diagnosis of Cushing's syndrome: an Endocrine Society Clinical Practice Guideline. J Clin Endocrinol Metab 2008;93:15261540.

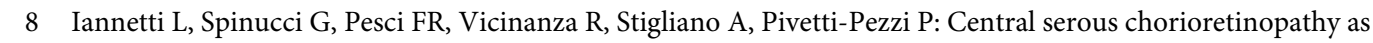
a presenting symptom of endogenous Cushing syndrome: a case report. Eur J Ophthalmol 2011;21:661-664. 